\title{
Morfología externa del desarrollo larvario de Hemicaranx amblyrhynchus (Pisces: Carangidae) del sur del Golfo de México
}

\author{
César Flores Coto ${ }^{1}$, Marina Sánchez Ramírez ${ }^{1}$, Faustino Zavala García ${ }^{1}$ \\ 1 Laboratorio de Zooplancton, Instituto de Ciencias del Mar y Limnología, Universidad Nacional Autónoma de México. \\ Apartado Postal 70-305. México D. F. 04510. México. Fax: 6160748. Coto@mar.icmyl.unam.mx
}

Recibido 30-VII-1997. Corregido 26-II-1998. Aceptado 3-III-1998.

\begin{abstract}
Larval development of Hemicaranx amblyrhynchus is described from wild specimens (standard length SL: 1.8-16.4 mm) caught in the southern Gulf of Mexico $(\mathrm{N}=25)$. A serrated occipital crest was present only in specimens smaller than $5.73 \mathrm{~mm} \mathrm{SL}$ and a supraocular crest with a small spine in those between 4 and $5 \mathrm{~mm}$. As in all larval carangids, the angle preopercle spine was conspicuous since the first stages. Supracleithral and posttemporal spines were present during flexion stage, and then disappeared in transformation stage. Body depth is moderate, increasing with development. The dorsal and anal fins start to be evident in the $2.65 \mathrm{~mm} \mathrm{NL}$ specimen; total number of spines and soft rays are almost complete around $9.0 \mathrm{~mm}$ SL. The pelvic bud was observed in the $3.16 \mathrm{~mm}$ LP specimens. From the early stages, the larvae are very pigmented and could be recognized by a pigmentation blotch on the tip of nose, which is conformed by palatine melanophores among others. Larvae of $H$. amblyrhynchus occurred scarcely in the area; they were essentially recorded on the inner shelf, in surface waters, not deeper than $30 \mathrm{~m}$. The present paper is the first larval record for the species in the southern Gulf of México.
\end{abstract}

Key words: Larval fishes, larval development, Hemicaranx amblyrhynchus, Gulf of Mexico.

Hemicaranx amblyrhynchus (Cuvier, 1833), conocida con el nombre común de casabe o chicharra, es una especie de hábitos costeros que penetra en aguas salobres; es más común a media agua y en el fondo que en la superficie, y no forma grandes cardúmenes (Berry y Smith-Vaniz 1978). Los organismos jóvenes se encuentran asociados con medusas (Nakamura 1980). Se distribuye en el Atlántico occidental, desde Carolina del Norte a Florianapolis (Brasil), es la única especie de este género en esta área (Berry y Smith-Vaniz 1978). En el sur del Golfo de México se presenta de manera muy escasa (Castro-Aguirre
1978, Reséndez-Medina 1981, Yáñez-Arancibia y Sánchez-Gil 1986).

Para la mayor parte de las especies de carángidos ya ha sido descrito su desarrollo larvario, así, de las 17 especies registradas en el sur del Golfo de México (Reséndez-Medina 1970, 1973, 1981, Castro-Aguirre 1978, Yáñez-Arancibia y Sánchez-Gil 1986), se ha descrito el desarrollo de 15 (Hildebrand y Cable 1930, McKenney et al. 1958, Berry 1959, Fields 1962, Aboussouan 1968, 1975, Conand y Franqueville 1973, Aprieto 1974, Montolio 1976, Tsokur 1977, Miller et al. 1979, Laro- 
che et al. 1984 y Sánchez-Ramírez y FloresCoto 1993). Sin embargo, algunas de estas descripciones están incompletas y faltan las de Decapterus tabl y $H$. amblyrhynchus, de esta última sólo se tiene el esquema de un organismo de $9.2 \mathrm{~mm}$ (Laroche et al. 1984), por lo que la descripción de su desarrollo larvario es el objeto del presente trabajo.

\section{MATERIALES Y METODOS}

El material empleado en este trabajo se recolectó en el sur del Golfo de México, en latitudes al sur de los $210 \mathrm{~N}$, en un área que abarca la zona oceánica y la plataforma continental de los estados de Veracruz, Tabasco y Campeche. La metodología de recolecta ha sido descrita por Flores-Coto y Sánchez-Ramírez (1989) y. Sánchez-Ramírez y Flores-Coto (1998).

La especie fue determinada a partir del ejemplar mas grande capturado (16.4 mm LP), el cual ya presenta las características merísticas completas de H. amblyrhynchus: DVIIVIII+I,27-29; A. II+I,23-25; vértebras 26 (Walls 1975, Hoese y Moore 1977, Fahay 1983). A partir de ese organismo se realizó una secuencia hacia tallas menores.

En la descripción del desarrollo larvario se consideraron la etapas de preflexión, flexión, postflexión y transformación, de acuerdo con lo señalado por Kendall et al. (1984).

En las larvas incluidas en la serie que se describe se determinaron sus características merísticas (espinas preoperculares, supracleitrales y postemporales, aleta dorsal, aleta anal, aleta caudal, aleta pélvica, fórmula preopercular) y morfométricas ( $\mathrm{LP}=$ longitud patrón, $\mathrm{LN}=$ longitud notocordal, $\mathrm{LHA}=$ longitud hocico-ano, $\mathrm{LC}=$ longitud de la cabeza, $\mathrm{AC}=$ altura del cuerpo y $\mathrm{DO}=$ diámetro del ojo), con una precisión de $0.01 \mathrm{~mm}$.
Se calcularon los porcentajes de la altura del cuerpo, la longitud hocico ano y la longitud de la cabeza con respecto a la longitud patrón, y el diámetro del ojo como porcentaje de la longitud de la cabeza.

La descripción del desarrollo se hizo con la precisión que permitió el material, por lo que la aparición y desaparición de estructuras a lo largo del desarrollo se refiere a los especímenes donde se hayan observado tales cambios.

\section{RESULTADOS}

Las larvas de esta especie fueron sumamente escasas en el área de estudio. A lo largo de 15 campañas oceanográficas, que comprendieron más de 550 muestras analizadas, se recolectaron únicamente 39 ejemplares (en las campañas de abril de 1983 y 1984, septiembre de 1992, febrero y agosto 1993), de los cuales 25 se incluyen en la serie que se describe, por ser los que se encontraban en mejores condiciones.

Las larvas de $H$. amblyrhynchus se presentaron sólo en aguas neríticas superficiales, hasta un máximo de $30 \mathrm{~m}$ de profundidad. En toda la plataforma se registraron especímenes en un amplio límite de tallas (1.63-16.43 mm LP); sin embargo, la mayor abundancia correspondió a ejemplares menores de $3 \mathrm{~mm}$ LP, esencialmente en áreas con profundidades menores de $20 \mathrm{~m}$.

Morfología (Figs. 1, 2, y 3): La larva más pequeña capturada que inicia la serie descrita, midió $1.87 \mathrm{~mm} \mathrm{LN}$, y estaba en estado de preflexión, en tanto el de mayor talla con 16.43 $\mathrm{mm}$ LP, ese encontró en proceso de transformación a juvenil. Los organismos entre 2.54 y $3.16 \mathrm{~mm}$ de LP se encuentran en proceso de flexión del urostilo (Cuadro 1).

El cuerpo es moderadamente alto en la preflexión (29.8\%), aumenta ligeramente a lo largo del desarrollo hasta $34.4 \%$ en la postflexión 


\section{CUADRO 1}

Características morfométricas (en $\mathrm{mm}$ ) y merísticas de larvas de Hemicaranx amblyrhynchus

\begin{tabular}{|c|c|c|c|c|c|c|c|c|c|c|}
\hline $\begin{array}{l}\text { Longitud } \\
\text { notocordal }\end{array}$ & $\begin{array}{l}\text { Longitud } \\
\text { patrón }\end{array}$ & $\begin{array}{l}\text { Longitud } \\
\text { hocico- } \\
\text { ano }\end{array}$ & $\begin{array}{l}\text { Longitud } \\
\text { de la } \\
\text { cabeza }\end{array}$ & $\begin{array}{c}\text { Altura } \\
\text { del } \\
\text { cuerpo }\end{array}$ & $\begin{array}{c}\text { Diámetro } \\
\text { del } \\
\text { ojo }\end{array}$ & $\begin{array}{l}\text { Aleta } \\
\text { dorsal }\end{array}$ & $\begin{array}{c}\text { Aleta } \\
\text { anal }\end{array}$ & $\begin{array}{c}\text { Aleta } \\
\text { pélvica }\end{array}$ & $\begin{array}{l}\text { Aleta } \\
\text { caudal }\end{array}$ & $\begin{array}{c}\text { Fórmula } \\
\text { preoper- } \\
\text { cular }\end{array}$ \\
\hline \multicolumn{11}{|l|}{ Preflexión } \\
\hline 1.87 & & 1.21 & 0.59 & 0.57 & 0.16 & - & - & - & - & $2+1+1$ \\
\hline 1.91 & & 1.11 & 0.66 & 0.57 & 0.20 & - & - & $\ldots$ & - & $2+1+1$ \\
\hline 1.99 & & 1.21 & 0.68 & 0.55 & 0.23 & - & - & - & - & $2+1+1$ \\
\hline 1.99 & & 1.15 & 0.62 & 0.55 & 0.20 & - & - & - & - & $2+1+1$ \\
\hline 2.05 & & 1.42 & 0.72 & 0.59 & 0.23 & - & - & - & - & $2+1+1$ \\
\hline 2.18 & & 1.42 & 0.82 & 0.66 & 0.27 & - & - & - & - & $2+1+1$ \\
\hline 2.34 & & 1.42 & 0.64 & 0.62 & 0.23 & - & - & - & - & $2+1+1$ \\
\hline 2.38 & & 1.56 & 0.94 & 0.78 & 0.27 & - & - & - & - & $2+1+1$ \\
\hline 2.50 & & 1.76 & 1.01 & 0.86 & 0.27 & - & - & - & - & $2+1+1$ \\
\hline \multicolumn{11}{|l|}{ Flexión } \\
\hline & 2.54 & 1.72 & 0.94 & 0.88 & 0.31 & 一 & - & - & - & $2+1+1$ \\
\hline & 2.65 & 1.81 & 1.05 & 0.86 & 0.31 & - & - & - & 一 & $2+1+1$ \\
\hline & 2.65 & 1.79 & 1.15 & 0.82 & 0.33 & - & - & 一 & - & $2+1+1$ \\
\hline & 2.73 & 1.83 & 1.09 & 0.90 & 0.31 & - & - & - & - & $2+1+1$ \\
\hline & 2.80 & 1.83 & 1.05 & 0.86 & 0.31 & - & - & - & - & $2+1+1$ \\
\hline & 2.83 & 1.76 & 1.09 & 0.90 & 0.31 & - & - & - & - & $2+1+1$ \\
\hline & 3.00 & 1.87 & 1.09 & 0.94 & 0.27 & - & - & - & - & $2+1+1$ \\
\hline & 3.04 & 1.97 & 1.13 & 1.03 & 0.27 & - & - & 一 & - & $2+1+1$ \\
\hline & 3.16 & 2.11 & 1.37 & 1.07 & 0.39 & - & - & - & - & $3+1+1$ \\
\hline \multicolumn{11}{|l|}{ Postflexión } \\
\hline & 4.34 & 2.89 & 1.82 & 1.48 & 0.49 & 21 & I, 15 & Presente & $0+8+7+0$ & $3+1+1$ \\
\hline & 4.37 & 2.95 & 1.72 & 1.54 & 0.49 & $\mathrm{~V}, 21$ & II, 15 & Presente & $0+9+8+0$ & $3+1+1$ \\
\hline & 5.05 & 3.39 & 2.03 & 1.79 & 0.55 & VI, 20 & II, 17 & Presente & $0+9+8+1$ & $4+1+2$ \\
\hline & 5.54 & 3.57 & 2.09 & 1.85 & 0.55 & VI, 22 & II+I, 18 & Presente & $0+9+8+0$ & $4+1+2$ \\
\hline & 5.73 & 3.70 & 2.22 & 1.97 & 0.59 & VII+I, 23 & II+I, 19 & Presente & $1+9+8+2$ & $4+1+2$ \\
\hline & 8.96 & 5.17 & 3.18 & 3.03 & 0.90 & VII+I, 26 & II+I, 23 & Presente & $7+9+8+7$ & $3+1+3$ \\
\hline \multicolumn{11}{|c|}{ Transformación } \\
\hline & 16.43 & 9.30 & 5.43 & 5.89 & 1.40 & VII+I, 28 & II+I, 25 & Presente & $9+9+8+8$ & $3+1+2$ \\
\hline
\end{tabular}

y alcanza $35.8 \%$ en el espécimen de $16.4 \mathrm{~mm}$ LP, que se encuentra en el final de la etapa de transformación. La proporción del ojo decrece desde $31.0 \%$ en preflexión a $27.3 \%$ en postflexión y $25.8 \%$ en la etapa de transformación.

Las proporciones de la longitud hocicoano y de la longitud de la cabeza, respecto a la longitud patrón, varían muy poco dentro de los límites de las tallas registradas; sin embargo, existe un ligero incremento de la preflexión a la postflexión (Cuadro 1).

La cresta supraoccipital que es aserrada, es visible desde la larva más pequeña de la se- rie $(1.87 \mathrm{~mm} \mathrm{LN})$ y aún se encuentra presente en la larva de $5.73 \mathrm{~mm} \mathrm{LP}$, aunque muy pequeña; después desaparece. La cresta supraocular que presenta una pequeña espina, se observa sólo en los ejemplares de 4.34, 4.37 y $5.05 \mathrm{~mm}$ de LP.

La espina angular del preopérculo es muy conspicua, particularmente en las primeras tallas. La fórmula preopercular es de $2+1+1$ a los $1.87 \mathrm{~mm} \mathrm{LN}$, a los $3.16 \mathrm{~mm}$ de LP aumenta a $3+1+1$, en organismos entre 5.05 y $5.73 \mathrm{~mm}$ de LP la fórmula es de $4+1+2$ y disminuye hasta tener $3+1+2$ a los $16.43 \mathrm{~mm}$ de LP (Cuadro 1). A los $2.54 \mathrm{~mm}$ LP aparece una espina supra- 
cleitral y una postemporal, mientras que a los $4.34 \mathrm{~mm}$ de LP hay dos supracleitrales. Ambos tipos de espinas tienden a desaparecer al aumentar la talla y en el ejemplar de $16.43 \mathrm{~mm}$ de LP ya no existen.

El brote de las aletas pélvicas se observó en la larva de $3.16 \mathrm{~mm}$ de LP. El desarrollo de las aletas dorsal y anal se inicia con un pequeño brote, alrededor de $2.65 \mathrm{~mm} \mathrm{LP}$; mientras que los primeras espinas y, o radios, tanto de estas aletas como de la caudal, se observan en el espécimen de $4.34 \mathrm{~mm}$ LP. En el organismo de $8.9 \mathrm{~mm}$ LP el número de espinas y radios de todas las aletas está casi completo y en el de $16.43 \mathrm{~mm}$ LP ya lo está (D. VII+I, 28; A. II+I, 25; C. $9+9+8+8)$, sin embargo, no podemos precisar la talla en la que se completa el número definitivo de radios y/o espinas debido a la falta de especímenes entre las dos tallas mencionadas (Cuadro 1).

Pigmentación (Figs. 1, 2, y 3): Esta especie presenta un patrón de característico desde la talla más pequeña registrada $(1.87 \mathrm{~mm}$ de LN). Dicho patrón consiste en una serie de melanóforos situados en la región lateral del cuerpo, siendo más evidentes los que se encuentran sobre la línea media. En la porción media dorsal del cuerpo hay una serie de melanóforos que se extiende desde el término de la cresta supraccipital hasta 2/3 de LP. También se encuentra otra serie de melanóforos en la porción media ventral, desde la sínfisis cleitral hasta el mismo nivel que la serie dorsal. Presenta melanóforos escasos sobre la cabeza, en el estómago y en la bóveda de la vejiga natatoria. Los pigmentos en el palatino que, aunados a otros externos en la punta de la mandíbula, maxila y alrededor de las aberturas nasales, conforman una gran mancha en la punta del hocico, que se extiende en dirección al ojo. Este patrón de pigmentación se conserva durante todo el desarrollo, aumentando en intensidad. a lo largo del mismo. Además, presenta una gran cantidad de pigmentos en todas las aletas, excepto en la aleta pectoral.

\section{DISCUSION}

La talla de eclosión de esta especie es menor de $1.87 \mathrm{~mm}$ de $\mathrm{LN}$, puesto que el ejemplar de esta talla, que es el más pequeño de los capturados no presenta ningún indicio de saco vitelino y tiene ojos ya totalmente pigmentados.

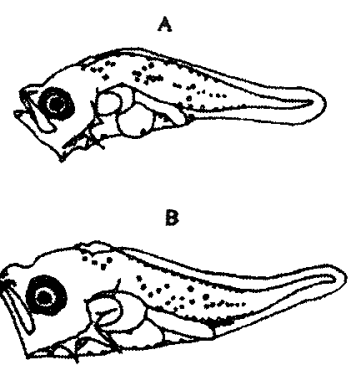

C

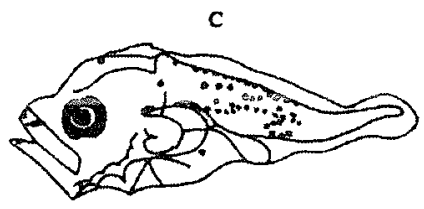

D

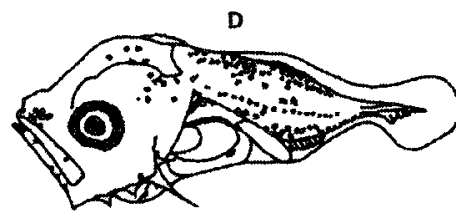

E

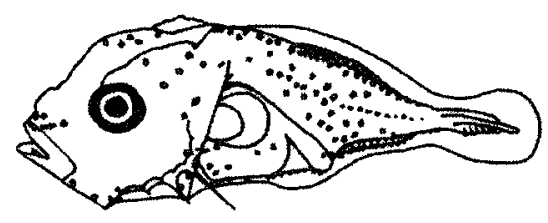

F

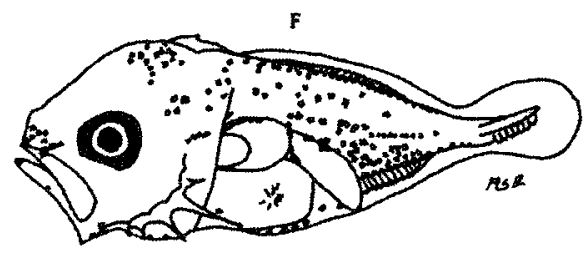

Fig. 1. Larvas de Hemicaranx amblyrhynchus en etapa de preflexión: A, $1.91 \mathrm{~mm}$; B, $2.18 \mathrm{~mm}$; C, $2.50 \mathrm{~mm}$ de LP, y flexión: D, $2.65 \mathrm{~mm}$; E, $3.00 \mathrm{~mm}$; F, $3.16 \mathrm{~mm}$ de LP. 
A

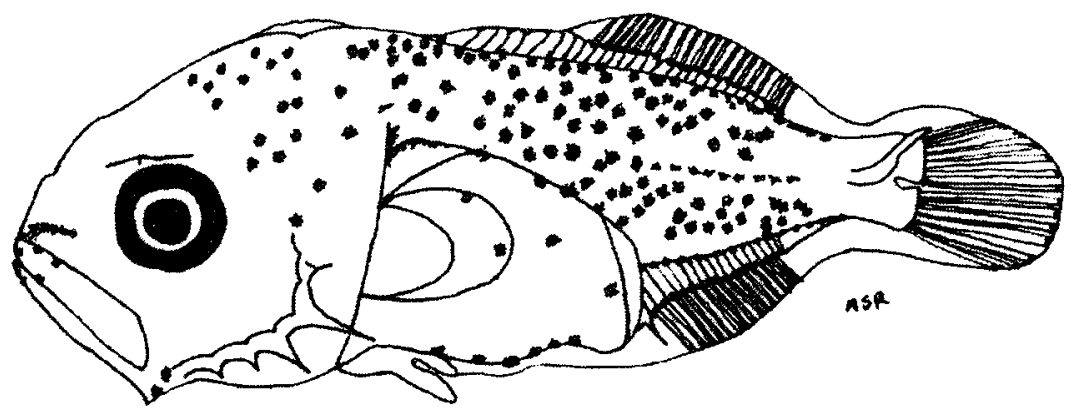

B

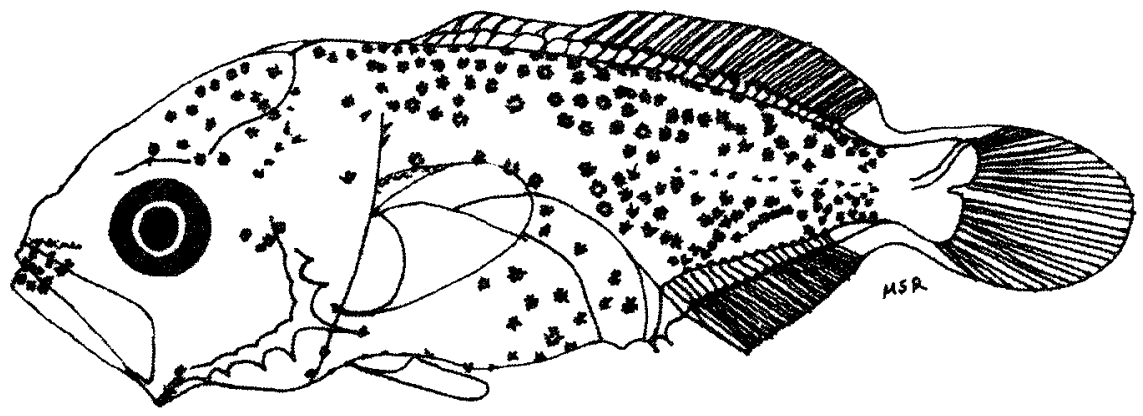

C

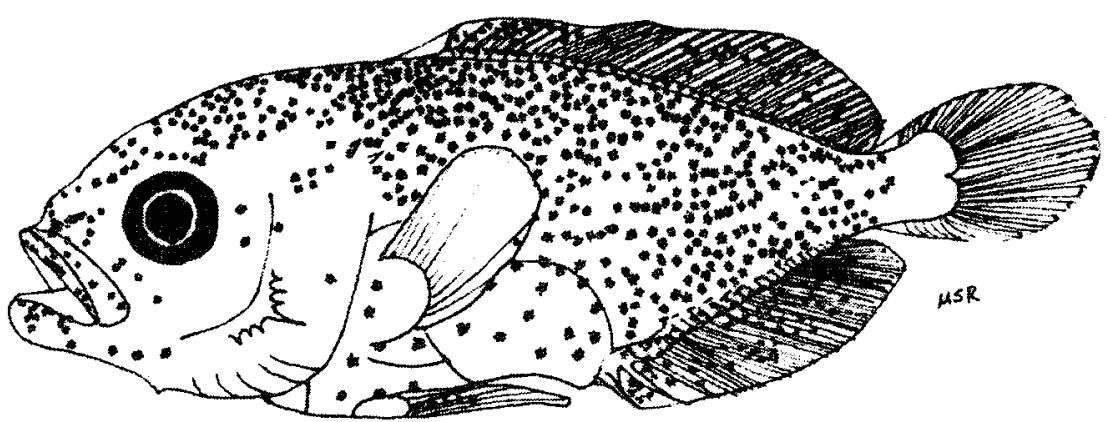

Fig. 2. Larvas de Hemicaranx amblyrhynchus en etapa de postflexión: A, 4.34 mm; B, 5.54 mm; C,8.96 mm de LP.

Las larvas de $H$. amblyrhynchus, cuando aún no tienen completos sus radios y espinas, pueden confundirse con las de Trachurus lathami; sin embargo, pueden diferenciarse, principalmente, por el pigmento en las mandíbulas y en el palatino, así como por la presencia de una cresta supraocular en $H$. amblyrhynchus. También es útil en la diferenciación el hecho que el proceso de flexión en $H$. amblirhynchus se presenta entre 2.4 y $3.16 \mathrm{~mm}$, en tanto en la otra especie es entre 4.0 y $5.0 \mathrm{~mm} \mathrm{LP}$. 


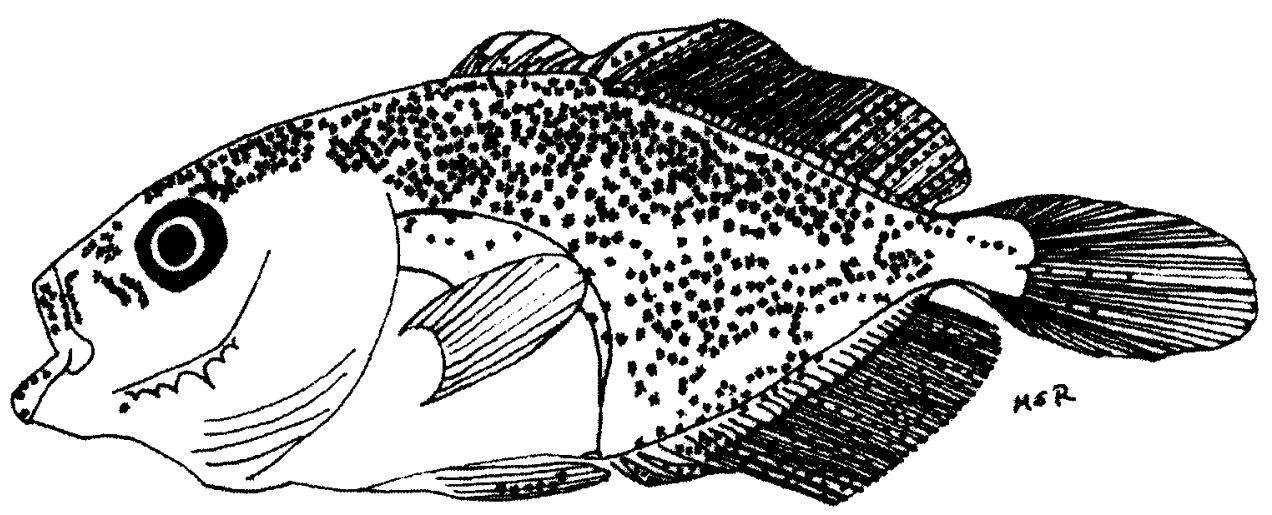

Fig. 3. Larva de Hemicaranx amblyrhynchus en etapa de transformación: 16.43 mm de LP.

\section{AGRADECIMIENTOS}

Los autores agradecen a la Dirección General de Asuntos del Personal Académico, UNAM (proyectos IN-202092 y IN203893) y al Consejo Nacional de Ciencia y Tecnología por su apoyo económico.

\section{RESUMEN}

Se describe el desarrollo larvario de Hemicaranx amblyrhynchus con especímenes capturados en el sur del Golfo de México. Los ejemplares presentaron tallas desde $1.87 \mathrm{~mm}$ hasta $16.43 \mathrm{~mm}$. Los ejemplares más pequeños presentan una cresta supraoccipital aserrada que se distingue todavía en el espécimen de $5.7 \mathrm{~mm}$ de LP y desaparece en los de talla superior. El cuerpo es moderadamente alto en la preflexión y aumenta ligeramente durante el desarrollo. Las aletas dorsal y anal son visibles a partir del espécimen de $2.65 \mathrm{~mm}$ de $\mathrm{LN}$; el número de espinas y radios está casi completo alrededor de los $9.0 \mathrm{~mm}$ LP. Las larvas de $H$. amblyrhynchus pueden reconocerse por una mancha de pigmentos en la punta del hocico, que está conformada, entre otros, por melanóforos en el palatino.

\section{REFERENCIAS}

Aboussouan, A. 1968. Eufs et larves de Téléostéens de l'Ouest africain. VI. Larves de Chloroscombrus chrysurus (Linné) et Blepharis crinitus (Mitchill) (Carangidae). Bull. Inst. Fondam. Afr. Noire, Sér. A Sci. Nat. 30: 226-237.

Aboussouan, A. 1975. EEufs et larves de Téléostéens de l'Ouest africain. XIII. Contribution à l'identification des larves de Carangidae. Bull. Inst. Fondam. Afr. Noire, Sér. A Sci. Nat. 37: 899-938.

Aprieto, V. L. 1974. Early development of five carangid fishes of the Gulf of Mexico and the South Atlantic coast of the United States. Fish. Bull. 72: 415-444.

Berry, F. F. 1959. Young jack crevalles (Caranx species) off the southeastern Atlantic coast of the United States. U. S. Fish. Wildl. Serv. Fish. Bull. 59: 417-535.

Berry, F. F. \& W. F. Smith-Vaniz. 1978. Carangidae. In W. Fischer (ed.). FAO species identification sheets for fisheries purposes Western Central Atlantic, Fishing Area 31. Vol. I-II. FAO, Roma.

Castro-Aguirre, J. L. 1978. Catálogo sistemático de los peces marinos que penetran a las aguas continentales de México con aspectos zoogeográficos y ecológicos. Dir. Gral. Inst. Nal. Pesca Serie Científica 19: 78-85

Conand, F. \& C. Franqueville. 1973. Identification et distribution saisonnière de larves de Carangides au large du Sénégal et de la Gambie. Bull. Inst. Fondam. Afr. Noire, Sér. A. Sci. Nat. 35: 951-978.

Fahay, M. P. 1983. Guide to the early stages of marine fishes ocurring in the western North Atlantic Ocean, Cape Hateras to the southern Scotian Shelf. J. Northw. Atl. Fish. Sci. 4: 1-432. 
Fields, H. M. 1962. Pampanos (Trachinotus spp.) of south Atlantic coast of the United States. U. S. Fish Wildl. Serv. Fish. Bull. 62: 189-222.

Flores-Coto, C. \& M. Sánchez-Ramírez. 1989. Larval distribution and abundance of Carangidae (Pisces), from the southern Gulf of Mexico. Gulf Res. Rep. 8: 117-128.

Hildebrand, S. F. \& L. E. Cable. 1930. Development and life history of fourteen teleostean fishes at Beaufort, N.C. U. S. Bull. Bur. Fish. 46: 383-488.

Hoese, H.D. \& R. H. Moore. 1977. Fishes of the Gulf of Mexico. (Texas, Louisiana and adjacent waters). Texas A \& M University. Houston, Texas. 327 p.

Kendall, Jr. A. W., E. H. Ahlstrom \& H. G. Moser. 1984. Early life history stages of fishes and their characters, p. 11-22. In Moser, H. G., W. J. Richardson, D. G. Cohen, M. P. Fahay, A. W. Kendall, Jr. \& S. L. Richardson (eds.). Ontogeny and Systematics of fishes. Spec. Publ. 1. Amer. Soc. Ichthyol. Herpetol., Allen, Lawrence, Kansas.

Laroche, W. A., W. F. Smith-Vaniz \& S. L. Richardson. 1984. Carangidae: development, p. 510-522. In Moser, H. G., W. J. Richardson, D. G. Cohen, M. P. Fahay, A. W. Kendall, Jr. \& S. L. Richardson (eds.). Ontogeny and Systematics of Fishes. Spec. Publ. 1. Amer. Soc. Ichthyol. Herpetol., Allen, Lawrence, Kansas.

McKenney, T. W., E. C. Alexander \& G. L. Voss. 1958. Early development and larval distribution of the carangid fish Caranx crysos (Mitchill). Bull. Mar. Sci. Gulf \& Caribb. 8: 167-200

Miller, J. M., W. Watson \& J. M. Leis. 1979. An atlas of common nearshore marine fish larvae of the Hawaiian islands. University of Hawaii, Honolulu, Oahu. 179 p.

Montolio, M. A. 1976. Estudio taxonómico y morfométrico de los estadios larvales de dos especies de Carangidae: Decapterus punctatus (Agassiz, 1829) y Caranx crysos (Mitchill, 1815) y su distribución en el Golfo de México. Rev. Invest. Inst. Nac. Pesca. 2: 85-125.
Nakamura E. L. 1980. Carangids of the northern Gulf of Mexico, p. 18-33. In M Flandorfer \& L. Skupien (eds.). Proceedings of a workshop for potential fishery resources of the northern Gulf of Mexico. MississippiAlabama.

Reséndez-Medina, A. 1970. Estudio de los peces de la Laguna de Tamiahua, Veracruz, México. An. Inst. Biol. Univ. Nal. Autón. México. Ser. Cienc. Mar Limnol. 4: 79-146.

Reséndez-Medina, A. 1973. Estudio de los peces de la Laguna de Alvarado, Veracruz, México. Rev. Soc. Mex. Hist. Nat. 34: 183-281.

Reséndez-Medina, A. 1981. Estudio de los peces de la Laguna de Términos, Campeche, México. II. Biótica 6: $345-430$

Sánchez-Ramírez, M. \& C. Flores-Coto. 1993. Desarrollo larvario y clave de identificación de algunas especies de la familia Carangidae (Pisces) del sur del Golfo de México. An. Inst. Cienc. Mar Limnol. Univ. Nal. Autón. México 20: 1-24.

Sánchez-Ramírez, M. \& C. Flores-Coto. 1998. Growth and mortality of larval Atlantic Bumper Chloroscombrus chrysurus (Pisces: Carangidae) in the southern Gulf of Mexico. Bull. Mar. Sci. (en prensa).

Tsokur, A. G. 1977. Ichthyoplankton of tropical waters of the world ocean. Larvae of carangid fishes (Carangidae, Pisces) of the Arabian Sea. Acad. Sci. U.S.S.R., Proc. P. P. Shirshov Inst. Oceanol. 109: 149-227.

Walls, J. G. 1975. Fishes of the northern Gulf of Mexico T. H. F., Nueva Jersey. 432 p.

Yáñez-Arancibia, A \& P. Sánchez-Gil. 1986. Los peces demersales de la plataforma continental del sur del Golfo de México. 1. Caracterización ambiental, ecología y evaluación de las especies, poblaciones y comunidades. An. Inst. Cienc. Mar Limnol. Univ. Nal Autón. México. Publ. Esp. 9: 1-230. 